\title{
In This Issue: Ultra-processed food and health
}

\author{
Allison Hodge
}

Back in 2009, Carlos Monteiro wrote a commentary for Public Health Nutrition describing three classes of foods from minimally to ultra-processed, and the association of ultra-processed foods with adverse health effects ${ }^{(1)}$. These classes were developed into the NOVA food classification, and many studies have since been conducted, showing the increasing consumption of ultra-processed foods and their association with many health outcomes, as well as with poorer dietary nutrient profiles ${ }^{(2)}$. The NOVA system was developed in Brazil and has been incorporated into the Brazilian Dietary Guidelines ${ }^{(3)}$; much of the research on NOVA food groups and ultra-processed foods has come from Brazil. In this issue, we include eight manuscripts describing studies of ultra-processed foods and other aspects of diet or health outcomes, seven of these were from Brazil and one from Spain.

Three studies were conducted in adults, covering gestational weight gain $^{(4)}$, cardiovascular events ${ }^{(5)}$ and blood pressure $^{(6)}$. In 259 pregnant women in Brazil, dietary intake was assessed by two 24-h recalls and the percentage of energy from ultra-processed foods calculated. During the third gestational trimester, an increment of $1 \%$ of energy from ultra-processed foods was associated with an additional weight gain of 4.17 (95\% CI 0.55, 7.79) g/week, which equates to an additional kilogram of weight gain over the 12 -week trimester in the 75th compared to the 25th percentile of ultraprocessed food intake ${ }^{(4)}$. Excess weight gain has potential adverse effects on infant and maternal health. Another cross-sectional study in 2359 cardiac patients from centres across Brazil looked at associations between the dietary inflammatory index (DII $\left.{ }^{\circledR}\right)$ and number of cardiovascular events or cardiometabolic risk factors, and intakes of different NOVA food categories. People in the top tertile of DII ${ }^{\circledR}$ had a higher risk of 2 or $\geq 3$ cardiovascular events and consumed more of all classes of processed foods, hence less of the unprocessed or minimally processed class (\% of energy $)^{(5)}$. In the ELSA-Brasil study, dietary intakes were assessed at baseline using FFQ and participants followed up around 4 years later for hypertension incidence and changes in blood pressure. Participants were 8754 civil servants from six cities in Brazil. Ultra-processed food provided around $25 \%$ of energy and intake was positively associated with risk of developing hypertension (OR 1.23 95\% CI 1.06, 1.44 for tertile $3 v$. tertile 1$)^{(6)}$.

The remaining five studies were in children ${ }^{(7-11)}$ or related to foods targeted to children ${ }^{(12)}$. In Spanish children with a mean age of 5.3 (SD 1.0) years, dietary intakes were collected using FFQ, foods classified according to the NOVA system and adherence to a Mediterranean style diet assessed by the KIDMED index. For each two-point increment in the KIDMED score (range -4 to +12 ), children consumed $3 \cdot 1 \%$ (95 \% CI 2.1, 4.0) less energy from ultraprocessed foods. Maintaining a traditional diet was identified as a way to avoid ultra-processed foods ${ }^{(7)}$. In southern Brazil, the daily frequency of consuming ultra-processed foods was associated with the risk of dental caries. More than two out of three children consumed ultra-processed foods four or more times per day; the prevalence ratios for non-cavitated (PR 2.25, $95 \%$ CI 1·19, 4.27) and cavitated caries (PR 3.48, $95 \%$ CI 1.18, 10.30) in this group was elevated relative to less frequent $\operatorname{consumption}^{(8,}$ 11). Marçal et $a l^{(9)}$ examined breast-feeding and ultra-processed food intake in Brazilian infants aged 6-24 months based on a single 24-h recall. These infants were from poor families participating in a cash transfer programme of government support. Of the 1604 infants studied, 223 (60.3\%) received some breast-feeding up to 1 year of age and $161(47 \cdot 6 \%)$ until 2 years of age ${ }^{(9)}$. Continuous breast-feeding was associated with less consumption of ultra-processed foods, of which the most consumed were biscuits, chocolate milk and baby food. The authors interpreted their results to recommend promotion of breast-feeding to minimise consumption of ultra-processed foods ${ }^{(9)}$. In another study of infants aged 6-24 months, Spaniol et al ${ }^{(10)}$ observed that ultra-processed foods made up around one-third of dietary energy intake, and infants consuming more processed and ultra-processed foods consumed more energy from saturated fat and sugar and more Na than lower ultra-processed food consumers ${ }^{(10)}$. Similar to the previous study from Brazil, the main ultra-processed foods consumed were infant and child food products, milk-sweetened beverages and bakery products ${ }^{(10)}$. The last of the studies considered was a survey of commercially available foods indicated for children under the age of 36 months from shops in Natal in Brazil $^{(12)}$. Across 100 retail stores, 1645 foods and/ beverages were examined but after excluding duplicates 95 different foods were included. Of these, $32 \%$ were breast milk substitutes and 26\% were cereals; $79 \%$ were ultraprocessed. All breast milk substitutes and follow-up formulas were classified as ultra-processed. Overall, ultra-processed foods had higher absolute values per 100 $\mathrm{g}$ for total and saturated fat, protein and carbohydrate and lower fibre than other foods. The median energy density of the ultra-processed foods was $1540 \mathrm{~kJ} / 100 \mathrm{~g}$ with 
$6.5 \%$ of total energy from proteins, $73.9 \%$ from carbohydrates and $19.6 \%$ from lipids ${ }^{(12)}$. These findings in infants and children point to the importance of health promotion and education to support breast-feeding and the use of minimally processed traditional foods.

This issue has reinforced the different ways an increasing reliance on ultra-processed foods can impact health from early life to adulthood. Also, we have five articles relating to the COVID-19 pandemic, which continues to impact on health and life globally.

\section{References}

1. Monteiro CA (2009) Nutrition and health. The issue is not food, nor nutrients, so much as processing. Public Health Nutr 12, 729-731.

2. Monteiro CA, Cannon G, Moubarac JC et al. (2018) The UN Decade of Nutrition, the NOVA food classification and the trouble with ultra-processing. Public Health Nutr 21, 5-17.

3. Brazilian Ministry of Health (2014) Dietary Guidelines for the Brazilian Population, [Mo Health, editor]. Brasília.

4. Gomes CB, Malta MB, Benicio MHD et al. (2020) Consumption of ultra-processed foods in the third gestational trimester and increased weight gain: a Brazilian cohort study. Public Health Nutr 1-9.

5. da Silva A, Felicio MB, Caldas APS et al. (2020) Pro-inflammatory diet is associated with a high number of cardiovascular events and ultra-processed foods consumption in patients in secondary care. Public Health Nutr: 1-10.

6. Scaranni P, Cardoso LO, Chor D et al. (2021) Ultra-processed foods, changes in blood pressure and incidence of hypertension: the Brazilian Longitudinal Study of Adult Health (ELSA-Brasil). Public Health Nutr 1-9.

7. da Rocha BRS, Rico-Campa A, Romanos-Nanclares A et al. (2020) Adherence to Mediterranean diet is inversely associated with the consumption of ultra-processed foods among Spanish children: the SENDO project. Public Health Nutr 1-10.

8. de Souza MS, Vaz JDS, Martins-Silva T et al. (2020) Ultraprocessed foods and early childhood caries in 0-3-year-olds enrolled at Primary Healthcare Centers in Southern Brazil. Public Health Nutr 1-9.

9. Marcal GM, Mendes MME, Fragoso M et al. (2020) Association between the consumption of ultra-processed foods and the practice of breast-feeding in children under 2 years of age who are beneficiaries of the conditional cash transfer programme, Bolsa Familia. Public Health Nutr 1-9.

10. Spaniol AM, da Costa THM, Souza AM et al. (2020) Early consumption of ultra-processed foods among children under 2 years old in Brazil. Public Health Nutr 1-11.

11. de Souza MS, Vaz JDS, Martins-Silva T et al. (2021) Ultraprocessed foods and early childhood caries in 0-3-year-olds enrolled at Primary Healthcare Centers in Southern Brazil ERRATUM. Public Health Nutr 24, 377.

12. da Rocha KF, de Araujo CR, de Morais IL et al. (2021) Commercial foods for infants under the age of 36 months: an assessment of the availability and nutrient profile of ultra-processed foods. Public Health Nutr 1-8. 